\title{
Research on Multiaircraft Cooperative Suppression Interference Array Based on an Improved Multiobjective Particle Swarm Optimization Algorithm
}

\author{
Huan Zhang, ${ }^{1}$ Rennong Yang, ${ }^{1}$ Changyue Sun, ${ }^{2}$ and Haiyan Han ${ }^{1}$ \\ ${ }^{1}$ Aeronautics and Astronautics Engineering College, Air Force Engineering University, Xian 710038, China \\ ${ }^{2}$ Tianjin University, Tianjin 300072, China \\ Correspondence should be addressed to Huan Zhang; scyl114@tju.edu.cn
}

Received 26 October 2016; Revised 11 January 2017; Accepted 17 January 2017; Published 5 March 2017

Academic Editor: Thomas Hanne

Copyright (C) 2017 Huan Zhang et al. This is an open access article distributed under the Creative Commons Attribution License, which permits unrestricted use, distribution, and reproduction in any medium, provided the original work is properly cited.

For the problem of multiaircraft cooperative suppression interference array (MACSIA) against the enemy air defense radar network in electronic warfare mission planning, firstly, the concept of route planning security zone is proposed and the solution to get the minimum width of security zone based on mathematical morphology is put forward. Secondly, the minimum width of security zone and the sum of the distance between each jamming aircraft and the center of radar network are regarded as objective function, and the multiobjective optimization model of MACSIA is built, and then an improved multiobjective particle swarm optimization algorithm is used to solve the model. The decomposition mechanism is adopted and the proportional distribution is used to maintain diversity of the new found nondominated solutions. Finally, the Pareto optimal solutions are analyzed by simulation, and the optimal MACSIA schemes of each jamming aircraft suppression against the enemy air defense radar network are obtained and verify that the built multiobjective optimization model is corrected. It also shows that the improved multiobjective particle swarm optimization algorithm for solving the problem of MACSIA is feasible and effective.

\section{Introduction}

Under the condition of information warfare, the enemy air defense radar network often has a strong ability to resist "the four" [1,2]. It increases the difficulty of combat aircraft penetration safely, so combat aircraft must have the aid of suppression interference against the enemy air defense radar network by the electronic support jamming aircraft. In this way it can provide a safe route planning space for subsequent route planning. Since the enemy air defense radar network is often deployed by a number of air defense radars, if only relying on a single electronic jamming aircraft to interfere with the enemy radar network, it is difficult to achieve the desired suppression effect due to the limited interference resources on a single jamming aircraft. Therefore, a solution of multiple electronic jamming aircraft cooperating with each other to interfere with the air defense radar network must be used. In order to make more reasonable distribution of interference resources in the process of cooperative interference, a reasonable array pattern should be used to determine the position of each jamming aircraft. This is the optimal problem of MACSIA in electronic warfare mission planning. For this problem, the domestic and foreign research are mainly focused on the operational efficiency and suppression interference effect of combat aircraft, and so on. But the study on the MACSIA is rare. In [3], Shi et al. analyzed the influence of electronic jamming on the path planning of combat aircraft. In [4], Wang et al. proposed a multiconstraint condition genetic algorithm to optimize the deployment of the enemy radar network. The calculation method of operational efficiency of combat aircraft against ground warning radar is studied under the condition of stand-off jamming [5]. Ruan et al. [6] have explored the influence of all kinds of factors on the suppression interference effect by taking the minimum interference distance as evaluation criterion. In a work by Tang et al. [7], the evaluation model of active 
suppression interference effect is built and the timing of interference is analyzed. In [8], Chen et al. have built a single objective optimal model of MACSIA, but the MACSIA is not considered as a multiobjective optimization problem (MOP) to solve. All the objective functions are aggregated into a single objective function by the weighted sum method, so a single objective optimization model for this problem is built. Different Pareto optimal solutions can be obtained by selecting different weight combinations. But the drawbacks of this method are obvious. Obviously the selection of weights is related to the relative importance of each objective function. If the user does not have sufficient prior knowledge of the problem, it is difficult to find the Pareto optimal solution that satisfies the decision maker. However, if the problem is regarded as a MOP to solve, it can avoid these drawbacks effectively.

In this paper, for the optimal problem of solving MACSIA, firstly, the calculation method of the enemy air defense radar detection range under the terrain masking condition is given, and the active interference model of electronic warfare is built. Secondly, the concept of route planning security zone is proposed and the mathematical morphology method is used to calculate the minimum width of route planning security zone. Thirdly, fully considering the characteristics of MOP for MACSIA, the minimum width of route planning security zone and the sum of the distance between each jamming aircraft and the center of the enemy air defense radar network are taken as objective functions, so the multiobjective optimization model of MACSIA is built. Finally, multiobjective particle swarm optimization (MOPSO) [9-17] has been widely used in multiobjective optimization based on its simple, fast convergence, easy to achieve in engineering [18-20], and so on. However, due to the disadvantages of particle swarm optimization algorithm [21], the distribution of nondominated solutions generated by MOPSO along the Pareto front is not very uniform and the computation time of MOPSO is not very fast enough. In order to solve these problems, the decomposition mechanism and the proportional distribution mechanism are introduced into the MOPSO algorithm. Therefore, an improved MOPSO algorithm is proposed which is used to solve the multiobjective optimization model of MACSIA. The optimal MACSIA schemes for the limit of the minimum width of route planning security zone and ensuring the safety of the jamming aircrafts are obtained by the simulation examples.

\section{Model Building}

\subsection{Radar Detection Model under the Terrain Masking Condition}

2.1.1. Digital Elevation Model (DEM). Digital elevation model (DEM) refers to a method of storing terrestrial elevation information in the form of specific data. There are two common representations of digital elevation models, which are the grid structure and the contour map. In this paper, the grid structure is used.

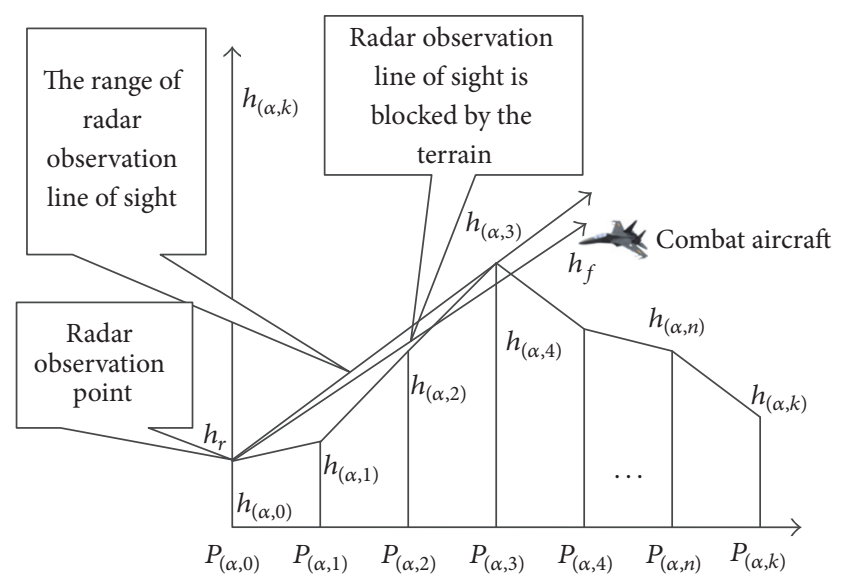

FIGURE 1: The calculation diagram of radar detection range under terrain masking condition.

2.1.2. Radar Detection Model. Radar detection model is generally characterized by radar equation which is defined as

$$
R=\left(\frac{P_{t} G^{2} \sigma \lambda^{2}}{(4 \pi)^{3}(S / N) F_{n} k T_{o} B_{n} L}\right)^{1 / 4} .
$$

In this equation, $R$ is the radar detection range $(\mathrm{m}), P_{t}$ is the radar emitting peak power $(\mathrm{W}), G$ is the radar antenna gain, $\sigma$ is the radar cross section $\left(\mathrm{m}^{2}\right), \lambda$ is the radar wavelength $(\mathrm{m})$, $S / N$ is the signal to noise ratio of radar receiver, $F_{n}$ is the noise coefficient, $k$ is the Boltzmann constant $\left(1.38 \times 10^{-23} \mathrm{~J} / \mathrm{K}\right)$, $T_{o}$ is the radar working temperature $(\mathrm{K}), B_{n}$ is the noise bandwidth $(\mathrm{Hz})$, and $L$ is the system loss.

\subsubsection{Calculating the Radar Detection Range under Terrain} Masking Condition. The calculation diagram of radar detection range under terrain masking condition is shown in Figure 1.

The height of the radar observation point is $h_{r}$. The height of the target point is $h_{f}$ and the target point represents the combat aircraft. The polar coordinate grid $P_{(\alpha, 3)}$ is the furthest boundary point of radar detection range in $\alpha$ direction and $h_{(\alpha, 3)}$ is the elevation value at this point.

In Figure 1, not only the height angle $\varepsilon_{P_{(\alpha, k)}}$ from the polar coordinate grid $P_{(\alpha, k)}$ to radar observation point in $\alpha$ direction can be calculated, but also the height angle $\varepsilon_{0}$ from the target point to radar observation point can be got, which are shown as

$$
\begin{aligned}
\varepsilon_{P_{(\alpha, k)}} & =\arctan \frac{h_{(\alpha, k)}-h_{r}}{P_{(\alpha, k)}-P_{(\alpha, 0)}}, \\
\varepsilon_{o} & =\arctan \frac{h_{f}-h_{r}}{P_{(\alpha, n)}-P_{(\alpha, 0)}} .
\end{aligned}
$$

Here, the values of $\varepsilon_{P_{(\alpha, k)}}(k=0,1,2, \ldots, k)$ are calculated in $\alpha$ direction. If there is a grid $P_{(\alpha, k)}$ satisfying $\varepsilon_{P_{(\alpha, k)}}$ is greater than $\varepsilon_{o}$; then the target point is within the shadow of the radar terrain and the radar could not detect the target. 
2.2. Active Interference Model of Electronic Warfare. The interference power expression of active electronic warfare equipment is defined as

$$
P_{r j}=\frac{P_{j} G_{j} G(\varphi) r_{j} B_{r}}{(4 \pi)^{2} R_{j}^{2} L_{j} B_{j}} .
$$

Here, $P_{j}$ is the jammer transmission power (W), $G_{j}$ is the jammer antenna gain, $G(\varphi)$ is the gain of the radar antenna in the jammer interference direction, $\varphi$ is the angle between the direction of the main lobe of the enemy radar antenna and jammer, $r_{j}$ is the jammer polarization loss, $B_{j}$ is the jammer interference signal bandwidth $(\mathrm{Hz}), B_{r}$ is the signal bandwidth of the enemy radar receiver $(\mathrm{Hz}), R_{j}$ is the distance between the enemy radar and the jammer $(\mathrm{m})$, and $L_{j}$ is the interference signal loss.

The power expression of the target echo signal is defined as

$$
P_{r t}=\frac{P_{t} G^{2} \sigma \lambda^{2}}{(4 \pi)^{3} R^{4} L}
$$

The meaning of each parameter in the above formula is shown in formula (1), where the constant $P_{r t}$ is equivalent to the parameter $S$ in formula (1).

The expression of $G(\varphi)$ is defined as

$$
G(\varphi)= \begin{cases}G, & 0 \leq|\varphi| \leq \varphi_{0.5} \\ K\left(2\left(\frac{\varphi_{0.5}}{\varphi}\right)\right)^{2} G, & \varphi_{0.5} \leq|\varphi| \leq 90^{\circ} \\ K\left(2\left(\frac{\varphi_{0.5}}{90^{\circ}}\right)\right)^{2} G, & 90^{\circ} \leq|\varphi| \leq 180^{\circ}\end{cases}
$$

In this equation, $\varphi_{0.5}$ is the lobe width of the enemy radar antenna at the half power point, $K$ is a constant between 0.04 and 0.1 in general, and $G$ is the enemy radar antenna gain.

The detection range expression of the enemy radar can be obtained from formulas (1), (3), and (4) in the case of interference with a single jammer, which is shown as

$R$

$$
=\left(\frac{P_{t} G^{2} \sigma \lambda^{2}}{(4 \pi)^{3} L F_{n} k T_{o} B_{n} K_{j}+4 \pi L P_{j} G_{j} G(\varphi) r_{j} B_{r} / B_{j}^{2} L_{j} B_{j}}\right)^{1 / 4} .
$$

Here, $K_{j}$ is the minimum suppression coefficient of the enemy radar and the remaining parameters are the same as in formulas (1), (3), and (4).

When considering suppression interference with multiple jammers against the enemy air defense radar network, the total interference power received is the sum of the interference powers of each jammer, which is defined as

$$
P_{r j \_t o t a l}=\sum_{i=1}^{n} \frac{P_{j i} G_{j i} G\left(\varphi_{i}\right) r_{j i} B_{r}}{(4 \pi)^{2} R_{j i}^{2} L_{j i} B_{j i}}
$$

The detection range expression of the enemy air defense radar after cooperative suppression by multiple jammers can be calculated, which is shown as

$$
R=\left(\frac{P_{t} G^{2} \sigma \lambda^{2}}{(4 \pi)^{3} L F_{n} k T_{o} B_{n} K_{j}+\sum_{i=1}^{n}\left(4 \pi L P_{j i} G_{j i} G\left(\varphi_{i}\right) r_{j i} B_{r} / B_{j i}^{2} L_{j i} B_{j i}\right)}\right)^{1 / 4}
$$

\subsection{Calculation Model of Route Planning Security Zone}

\subsubsection{Concept of Route Planning Security Zone. Electronic} jamming aircraft implements active suppression interference against the enemy air defense radar network, which aims to suppress the detection range of the enemy air defense radar and expand the space scope of route planning security. So it can provide more safe and reliable planning space for subsequent route planning. The definition of route planning security zone is given here. Route planning security zone is a certain width, height range for combat aircraft flight track space which constitutes the search space for the subsequent optimal route planning of combat aircraft. For the sake of convenience, this paper mainly calculates the width of route planning security zone for combat aircraft in a certain altitude. This width refers to the minimum width within the entire security zone.
2.3.2. Calculating the Width of Route Planning Security Zone Based on Mathematical Morphology. Since the detection range of the enemy air defense radar is influenced by the terrain, the detection boundary is irregular, so the model building is more difficult. If the traditional geometric method is used to calculate the width of security zone, the calculation process will be very complex and not conducive to engineering practice. Therefore, this paper will proceed from the mathematical morphology point of view so as to calculate the width of route planning security zone. Here the brief introduction to mathematical morphology will be given.

The core idea of mathematical morphology is to use a probe structure element to detect a given image and gets the information about the image, so the image can be analyzed and processed [22]. Mathematical morphology involves the basic operation of a closed operation, open operation, corrosion, expansion, and so on. The effect of the corrosion and 
expansion is opposite. The corrosion can cause a given area to shrink inward at the same time while the expansion allows the region to expand around. Both the closed operation and open operation are complexes of corrosion and expansion. The closed operation expands the image firstly and then corrodes. As a result, a narrow fracture on a given image can be filled and the holes in the image can be removed, and so on. On the contrary, the open operation corrodes the image firstly and then expands, which usually has a smoothing effect on a given image contour and eliminates small burrs on the contour.

This paper will be followed by the principle of image expansion and corrosion. Firstly, the image of radar detection range under terrain masking and electronic interference is binarized [22]. Secondly, we will do open operation on the binary image and then expand it. Thirdly, continuity check is performed while the image is being expanded and then the number of the graphic elements in the image is obtained. Finally, it is judged whether the route planning security zone satisfying the minimum width restriction is formed, and then the minimum width of security zone is calculated. The specific calculation process is shown in Figure 2.

2.4. Multiobjective Optimization Model of MACSIA. A typical maximization MOP can be defined as follows [23]:

$$
\begin{array}{ll}
\max & {\left[f_{1}(x), f_{2}(x), \ldots, f_{n}(x)\right]} \\
\text { s.t. } & \mathrm{lb} \leq x \leq \mathrm{ub} \\
& \text { Aeq } * x=\text { beq } \\
& A * x \leq b .
\end{array}
$$

In this equation, $n$ is the dimension of objective space, $x \in$ $\left[x_{1}, x_{2}, \ldots, x_{\text {Dim }}\right]^{T}$ is decision variable, Dim represents the number of decision variables, $\mathrm{ub}$ and $\mathrm{lb}$ are the upper and lower bounds of the decision variable $x$, respectively, and $A * x \leq b$ and $A$ eq $* x=b$ eq are linear inequality constraints and equality constraints for decision variables, respectively.

The ultimate goal of optimizing the MACSIA is to achieve a reasonable distribution of jamming aircraft positions and suppress the best against the enemy air defense radar, and the jamming aircraft themselves are not threatened. Therefore, this problem is a MOP. The expressions of single objective are defined as

$$
\begin{aligned}
& f_{1}=\sum_{i=1}^{N_{\text {radar }}}\left(\left(x_{i}-x_{t c}\right)^{2}+\left(y_{i}-y_{t c}\right)^{2}+\left(z_{i}-z_{t c}\right)^{2}\right)^{1 / 2} \\
& f_{2}=\text { Width }_{\text {safe }} .
\end{aligned}
$$

Here, the objective function $f_{1}$ represents the sum of the distances between our jamming aircrafts and the center of the enemy radar network, and the physical meaning is our own safety of jamming aircrafts. The objective function $f_{2}$ represents the product of the quantity and width of route planning security zone, and the physical meaning is the interference suppression effect of our jamming aircrafts against the enemy air defense radar network. $N_{\text {radar }}$ is the number of the enemy radars. $\left(x_{i}, y_{i}, z_{i}\right)$ represents the coordinates of the $i$ th

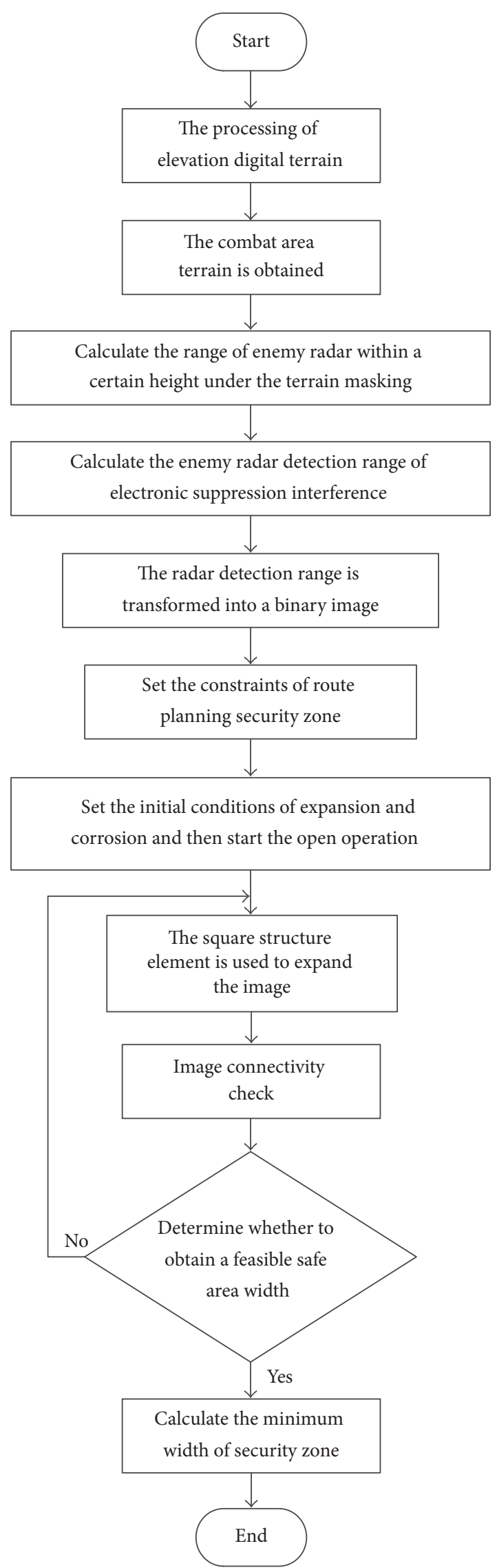

FIGURE 2: The calculation process of route planning security zone. 
jamming aircraft, $\left(x_{t c}, y_{t c}, z_{t c}\right)$ is the center coordinates of the enemy radar network, and Width safe $_{\text {is }}$ is the minimum width of the security zone.

The following constraints and interference principles should be considered when the MACSIA is implemented. Firstly, the position of each jamming aircraft must be outside the maximum detection range of the enemy radar. Secondly, the height of each jamming aircraft should be within the given safe height range. Thirdly, the interference principle is traditional principle of many-to-one and one-to-one. The constraints are mathematically expressed as follows:

$$
\begin{array}{ll}
\text { s.t. } & \operatorname{sqrt}\left(\left(x_{i}-x_{t j}\right)^{2}+\left(y_{i}-y_{t j}\right)^{2}+\left(z_{i}-z_{t j}\right)^{2}\right) \\
& >R_{j} \\
& \left(i=1,2, \ldots, N_{\mathrm{ECM}}, j=1,2, \ldots, N_{\text {radar }}\right) \\
& z_{\min } \leq z_{i} \leq z_{\max } .
\end{array}
$$

Here, $z_{\max }$ and $z_{\min }$ are the upper and lower bounds of the given height range, respectively. The parameter $\left(x_{i}, y_{i}, z_{i}\right)$ can be seen in (10), $N_{\mathrm{ECM}}$ is the quantity of the jamming aircrafts, $\left(x_{t j}, y_{t j}, z_{t j}\right)$ represents the coordinates of the $i$ th enemy radar, $N_{\text {radar }}$ is the quantity of the enemy radar, and $R_{j}$ is the maximum detection range of the $i$ th enemy radar.

The multiobjective optimization model of MACSIA with constraints is built by formulas (10), (11), and (12).

\section{The Improved MOPSO Algorithm}

3.1. Particle Swarm Optimization. Particle swarm optimization [24] is mainly used to solve single objective optimization problem. The particle position and velocity updating formulas of particle swarm optimization are defined as

$$
\begin{aligned}
& X_{i+1}=X_{i}+V_{i+1} m, \\
& V_{i+1}=w V_{i}+c_{1} r_{1}\left(\text { Pbest }_{i}-X_{i}\right)+c_{2} r_{2}\left(\text { Gbest }_{i}-X_{i}\right),
\end{aligned}
$$

where $X_{i+1}$ is the position of the particle at the next moment and $X_{i}$ represents the current position of the particle. $V_{i+1}$ is the velocity of the particle at the next moment, $V_{i}$ represents the current velocity of the particle. $c_{1}$ and $c_{2}$ are learning factors. $r_{1}$ and $r_{2}$ are random numbers with values between 0 and 1 . Pbest $t_{i}$ represents the individual optimal solution of the $i$ th particle and Gbest $t_{i}$ represents the global optimal solution of the $i$ th population. $w$ is inertia weighting factor which is defined as

$$
w=w_{\max }-\frac{w_{\max }-w_{\min }}{\text { itera }_{\max }} \text { itera. }
$$

Here, $w_{\max }$ and $w_{\min }$ are the maximum and minimum values of inertia weighting factors, respectively, itera is the current population iteration number, and itera $a_{\max }$ represents the maximum number of population iterations.

3.2. MOPSO Algorithm. MOPSO [25-30] algorithm uses multiple objective functions as the optimization functions and these objective functions are optimized to achieve the best state at the same time. The core idea of MOPSO can be described as follows: the particle population is initialized. Based on the idea of domination for each particle objective function value, the initial population is divided into two parts: the dominant subset $A$ and the nondominant subset $B$. An external file is used to store the nondominated solution subset generated by each iteration and only the particle position and velocity in the dominant subset $A$ are updated during the iteration. The dominance relation between the updated particles in $A$ and $B$ is compared. If $x_{i}$ belongs to $A, x_{j}$ belongs to $B$, and $x_{i}$ dominates $x_{j}$, then $x_{j}$ is rejected, $x_{i}$ is added to $B$, and the external archive is updated. The end of the algorithm can be predefined by maximum number of iterations, calculation accuracy, and so on. When the algorithm is finished, the nondominated solution set is the Pareto optimal solution.

The flow chart of MOPSO algorithm is shown in Figure 3.

\subsection{The Improved MOPSO Algorithm}

3.3.1. Decomposition Mechanism. The decomposition mechanism decomposes a MOP into a number of scalar optimization subproblems and optimizes them simultaneously. Each subproblem is optimized by only using information from its several neighboring subproblems, so it can reduce the computational complexity of the algorithm effectively. In this proposed algorithm, the adopted decomposition mechanism is classical Tchebycheff Approach [31], which is written in the form as follows:

$$
\begin{gathered}
g^{t e}\left(\frac{x}{\lambda}, z^{*}\right)=\max _{1 \leq i \leq n} \quad\left\{\lambda_{i}\left|f_{i}(x)-z_{i}^{*}\right|\right\} \\
\text { subject to } \quad x \in \Omega
\end{gathered}
$$

where $\lambda=\left(\lambda_{1}, \ldots, \lambda_{n}\right)^{T}$ is a weight vector and $z^{*}=\left(z_{1}^{*}, \ldots, z_{n}^{*}\right)^{T}$ is the reference point; that is, $z_{i}^{*}=\max \left\{f_{i}(x), x \in \Omega\right\}$ for each $i=1, \ldots, n$.

3.3.2. Proportional Distribution Mechanism. In the MOPSO algorithm, the selection of appropriate local guides from the new found nondominated solutions which aims to attain both convergence and diversity of solutions is a vital problem. If there are only a few nondominated solutions consisted in an area, more particles should be distributed to follow these nondominated solutions as a guide for searching larger variety of solutions around this area. If the situation is reversed, less particles should be distributed to avoid similar solutions gathered in this region. To maintain the diversity of new found nondominated solutions and improve the search ability of population, the proportional distribution mechanism is introduced. The proportional distribution mechanism is depicted as Figure 4 [32].

In Figure 4, the circles represent the particles and the triangles are the nondominated solutions. Each particle and nondominated solution is numbered. The distance between two adjacent nondominated solutions is defined as the variable $d$. The process of proportional distributed mechanism for the nondominated solutions can be described as follows. 


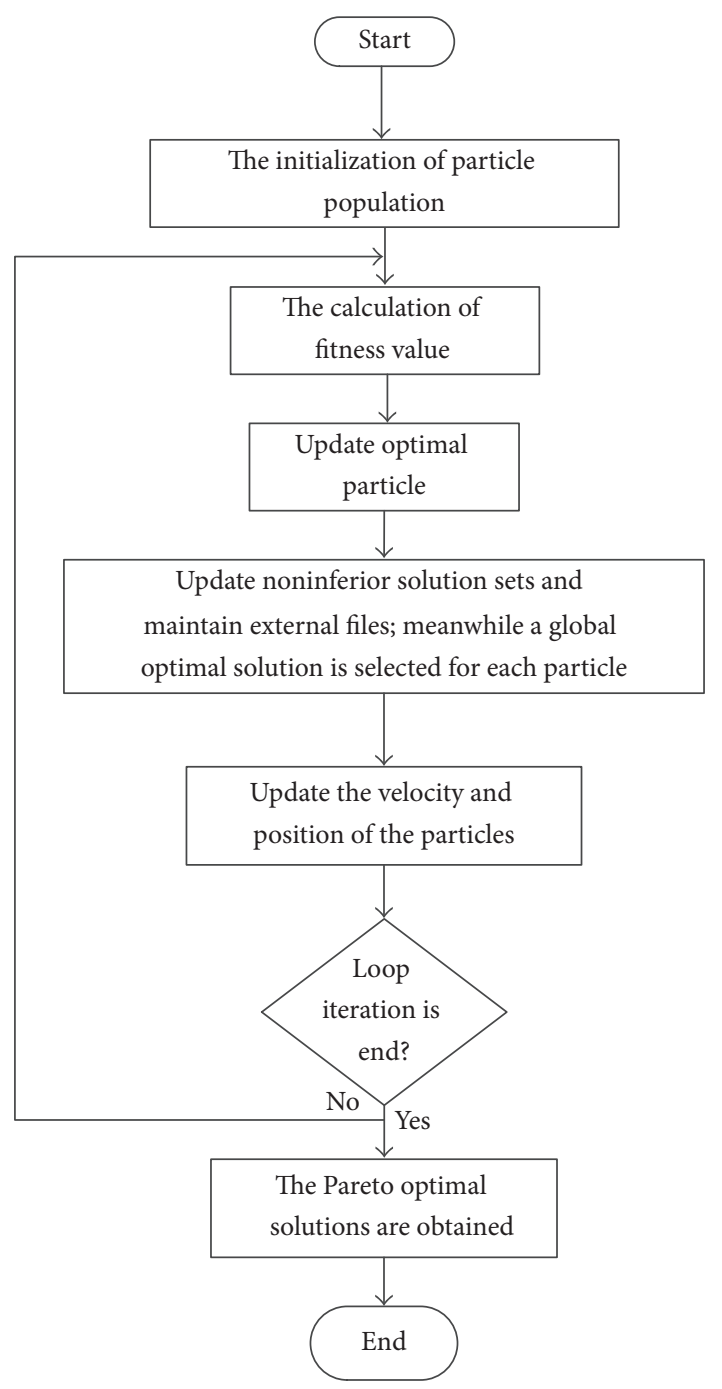

FIGURE 3: The flow chart of MOPSO.

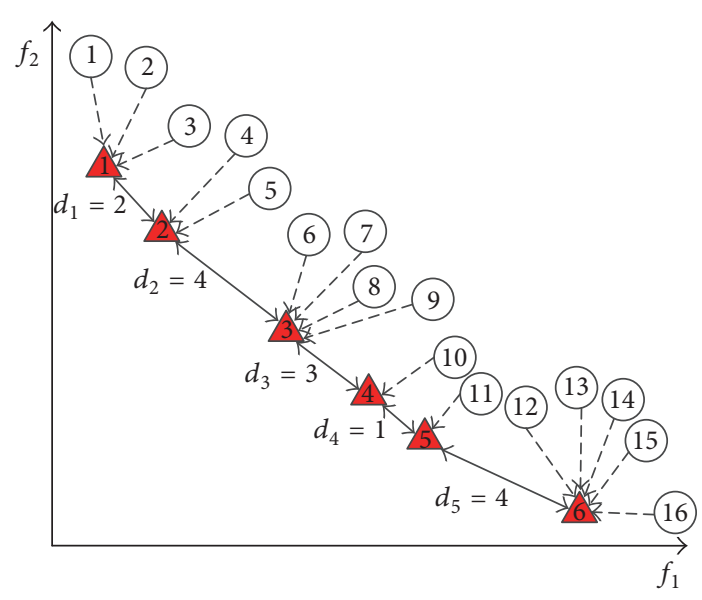

FIGURE 4: Local guides distribution for each particle.

Firstly, the existing nondominated solutions and all particles should be given a sorted number based on lateral axial.
The number results are shown as Figure 4. Secondly, calculate the density parameter $C_{i}$ of each nondominated solution in the solution space. The formula of $C_{i}$ is defined as

$$
\begin{aligned}
& C_{i} \\
& \quad= \begin{cases}\frac{\left[d\left(x_{i}, x_{i+1}\right)+d\left(x_{i}, x_{i-1}\right)\right]}{2}, & \text { if } i \neq n_{P F}, i \neq 1, \\
d\left(x_{i}, x_{i+1}\right), & \text { if } i=1 \\
d\left(x_{i}, x_{i-1}\right), & \text { if } i=n_{P F},\end{cases}
\end{aligned}
$$

where $x_{i}$ is the coordinate of nondominated solution $i$ and $n_{P F}$ is the total number of nondominated solutions obtained.

Thirdly, each nondominated solution is regarded as the guide distribution of particles. How the amount of particles is guided by each nondominated solution calculated is shown in (17), which is described as Gbest $N_{i}$.

$$
\text { Gbest } N_{i}=\operatorname{integer}\left(\frac{C_{i} * N_{p}}{\sum_{i=1}^{n} C_{i}}\right),
$$

where $N_{p}$ is the total number of particles and $C_{i}$ is defined as (16). The example calculation result of Gbest $N_{2}$ is $2 * 16 /(2+$ $3+3.5+1+1+4)=2$. The result indicates that three particles of numbers 1,2 , and 3 are guided by the nondominated solution 1 during the evolution process. In the proportional distribution mechanism, the sum of all Gbest $N_{i}$ which are defined as $N_{\text {Gbest }}$ should be equal to $N_{p}$ for the evolution process. Finally, the particles are guided by the nondominated solutions based on formula (17) and the guided result can be seen in Figure 4.

The decomposition mechanism and the proportion distribution mechanism are joined in the MOPSO algorithm flow, which constitutes the calculation process of the improved MOPSO algorithm. Based on the calculation process of the improved MOPSO algorithm, the multiobjective optimization model of MACSIA established in Section 2.4 is taken as the objective functions and then combined with the minimum width calculation method of route planning security zone in Section 2.3.2 which can be seen in Figure 2. Therefore, the MACSIA schemes can be obtained that meet the minimum width restriction of route planning security zone and the jamming aircrafts themselves are the most secure.

\section{Experiment Analysis}

$432 \mathrm{~km} \times 432 \mathrm{~km}$ area is selected as combat scenario in our simulation experiment. The DEM of the grid structure in this area is shown in Figure 5. The DEM data resolution is $360 \mathrm{~m}$. We assume that four enemy air defense radars are deployed in the area. The minimum suppression coefficient $K_{j}$ of the four radars is five. Their performance parameters are shown in Table 1 . The coordinates of the four radars are $(240 \mathrm{~km}, 100 \mathrm{~km}),(150 \mathrm{~km}, 120 \mathrm{~km}),(300 \mathrm{~km}, 250 \mathrm{~km})$, and $(180 \mathrm{~km}, 280 \mathrm{~km})$, respectively. In order to obtain at least $20 \mathrm{~km}$ width of the route planning security zone, we use three electronic jamming aircrafts to implement cooperative 
TABLE 1: The performance parameters list of four air defense radars.

\begin{tabular}{lccccccc}
\hline Radar & $P_{t} / \mathrm{kW}$ & $G$ & $\lambda / \mathrm{m}$ & $L$ & $F_{n}$ & $T_{o} / \mathrm{K}$ & $B_{n} / \mathrm{Hz}$ \\
\hline Number 1 & 4000 & 50 & 0.6 & 2.5 & 3 & $2 \times 1$ & 2.5 \\
Number 2 & 3900 & 45 & 0.8 & 4 & 3 & 4 & $2.1 \times 10^{5}$ \\
Number 3 & 3100 & 70 & 0.75 & 3 & 6 & 291 & 291 \\
Number 4 & 2600 & 100 & 0.5 & & $30^{5}$ & $1.5 \times 10^{5}$ \\
\hline
\end{tabular}

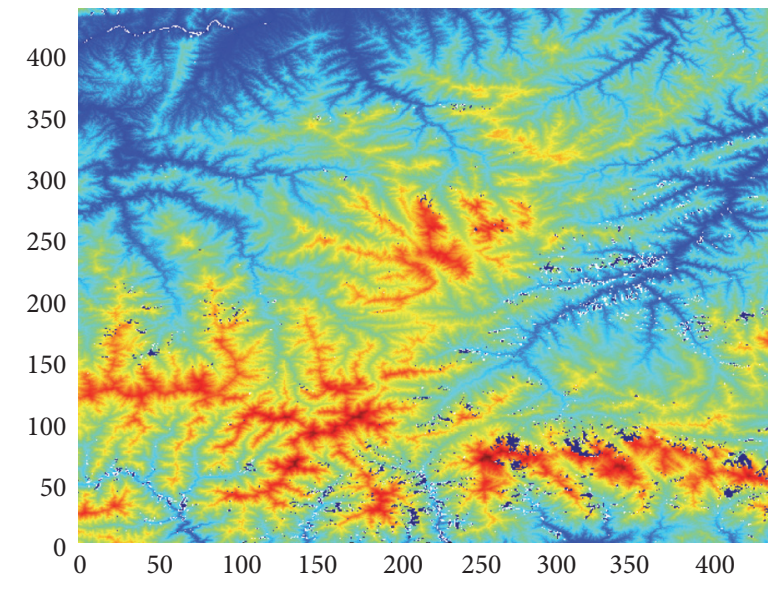

FIgURE 5: Digital elevation terrain map.

TABLE 2: The parameters list of three jamming aircrafts.

\begin{tabular}{lccccc}
\hline Jamming aircraft & $P_{j} / \mathrm{kW}$ & $G_{j}$ & $r_{j}$ & $L_{j}$ & $B_{j} / \mathrm{Hz}$ \\
\hline Number 1 & $1 \times 10^{3}$ & 4 & 2 & 20 & $2 \times 10^{6}$ \\
Number 2 & $2 \times 10^{3}$ & 2 & 2 & 20 & $2 \times 10^{6}$ \\
Number 3 & $2 \times 10^{3}$ & 6 & 1 & 30 & $2 \times 10^{6}$ \\
\hline
\end{tabular}

active suppression interference against the enemy radars. The three jamming aircrafts are at a height of $2.1 \mathrm{~km}$ and their performance parameters are shown in Table 2. The interference principle of one-to-one is used to interfere with the enemy radars.

Combined with the above method of calculating the radar detection range under the terrain masking condition, the detection range of $2100 \mathrm{~m}$ height for the four enemy air defense radars under the terrain masking condition is shown in Figure 6. It can be seen from Figure 6 that the four radars detection range overlaps each other. So it is difficult for our combat aircraft to penetrate in the past safely. Therefore, our combat aircraft must have the aid of suppression interference against air defense radar network by the electronic support jamming aircraft. The binary image of the radar network detection range is shown in Figure 7.

In order to facilitate the comparison of the MOPSO algorithm and the improved MOPSO algorithm, the initial parameters of the two algorithms are the same which are set as follows: $\operatorname{Dim}=6$, the population number $x$ Size is 50, the maximum number of iterations Max itera is 100, the learning factors $c_{1}$ and $c_{2}$ are both 0.8 , and the maximum value $w$ max and the minimum value $w$ min of the inertial

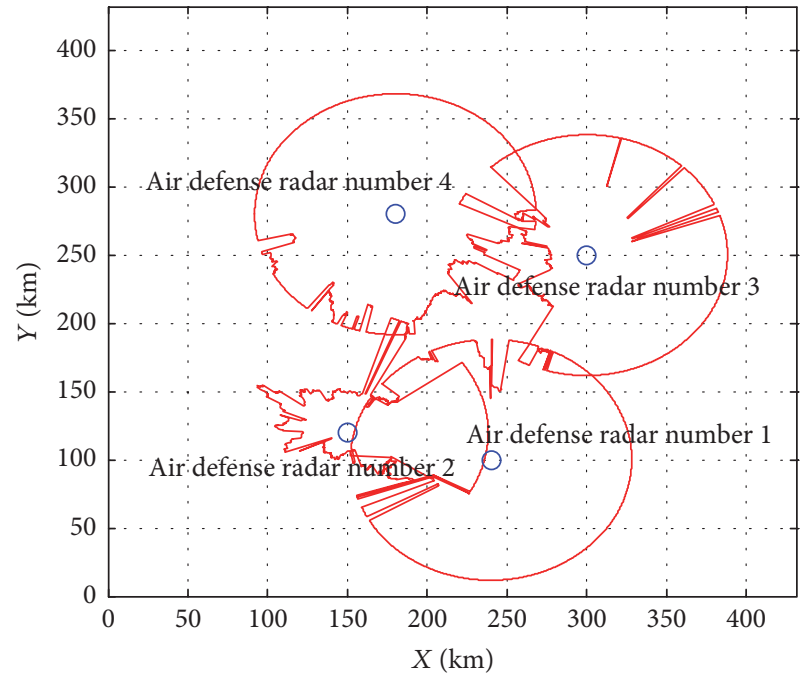

FIgURE 6: The detection range of the four enemy air defense radars under the terrain masking condition.

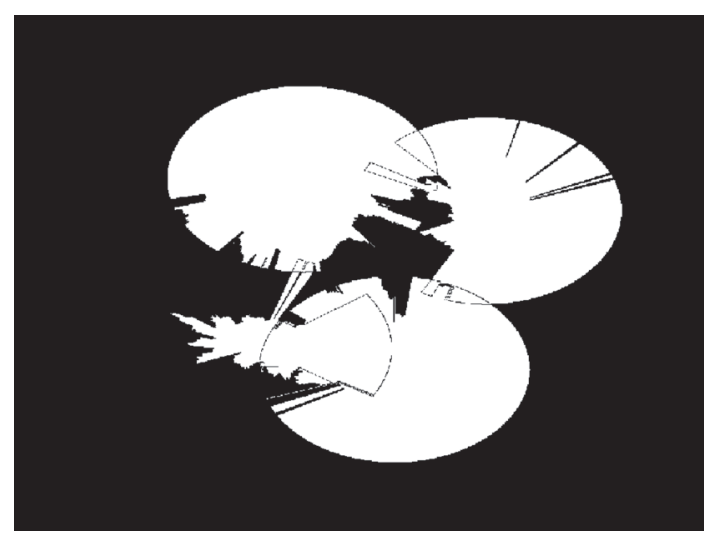

FIGURE 7: The binary image of radar network detection range.

weighting factors are 1.2 and 0.1 , respectively. The maximum number of iterations Max_itera is 100.

The simulation experiment is carried out by MATLAB language and the nondominated solutions distribution obtained is shown in Figure 8.

It can be seen from Figure 8 that the nondominated solutions obtained by the improved MOPSO algorithm obviously dominate the results of MOPSO, and the distribution of the nondominated solutions obtained by the improved MOPSO is more uniform. The number of the nondominated solutions obtained by MOPSO is 17 and the number of the 


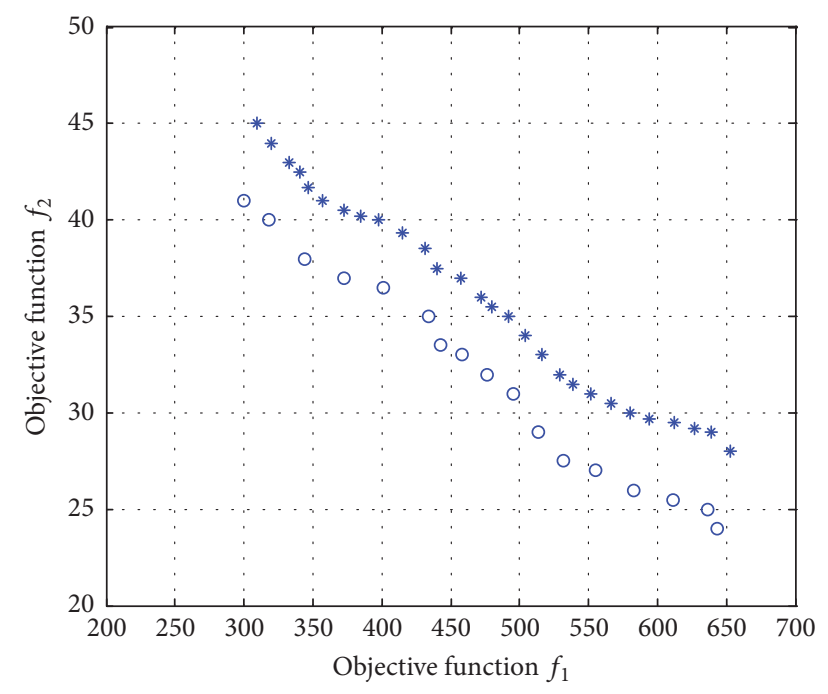

- The nondominated solution obtained by MOPSO

* The nondominated solution obtained by improved MOPSO

FIGURE 8: The distribution graph of nondominated solutions obtained.

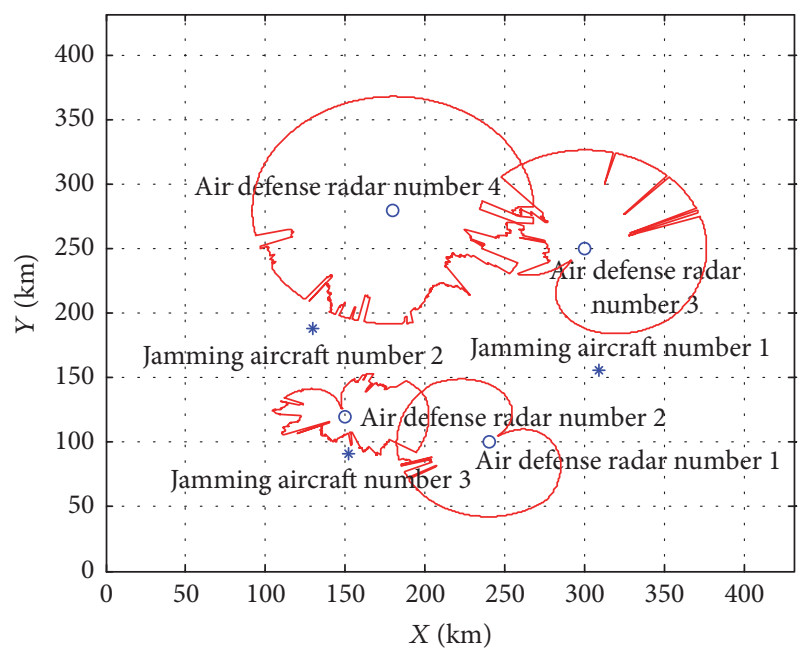

FIgURE 9: The optimal MACSIA scheme of the jamming aircrafts under the minimum total distance and maximum security zone minimum width obtained by MOPSO.

nondominated solutions obtained by the improved MOPSO is 28, which constitute their Pareto front, respectively. It is obvious that the Pareto front obtained by the improved MOPSO is more smooth than that of MOPSO. So the improved MOPSO algorithm has achieved a good search result than MOPSO.

For the improved MOPSO algorithm, there are 28 kinds of MACSIA schemes for the jamming aircrafts from the 28 Pareto optimal solutions. Which solution will be chosen requires the decision maker to make a choice based on the actual battlefield situation. Three typical cases are given here. Firstly, when the decision maker needs the sum of the distance between each jamming aircraft and the center of

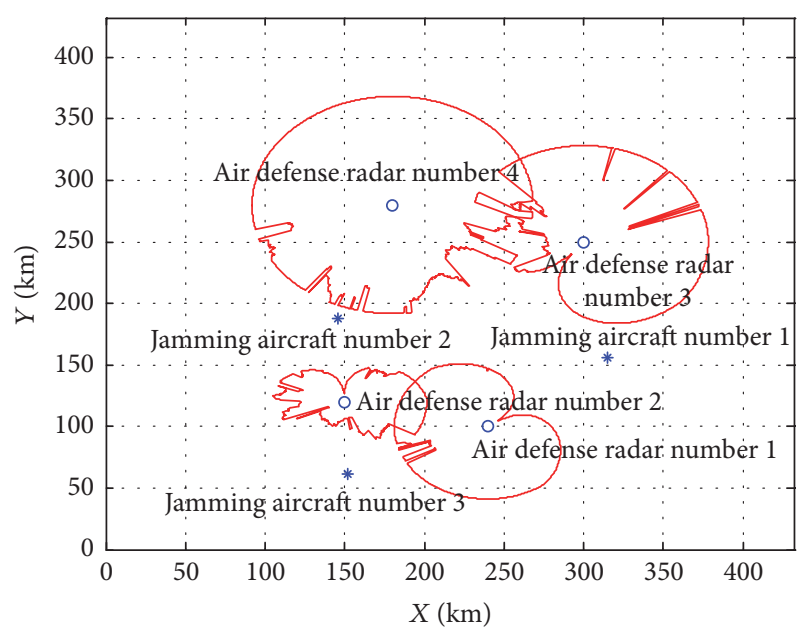

FIGURE 10: The optimal MACSIA scheme of the jamming aircrafts under the minimum total distance and maximum security zone minimum width obtained by improved MOPSO.

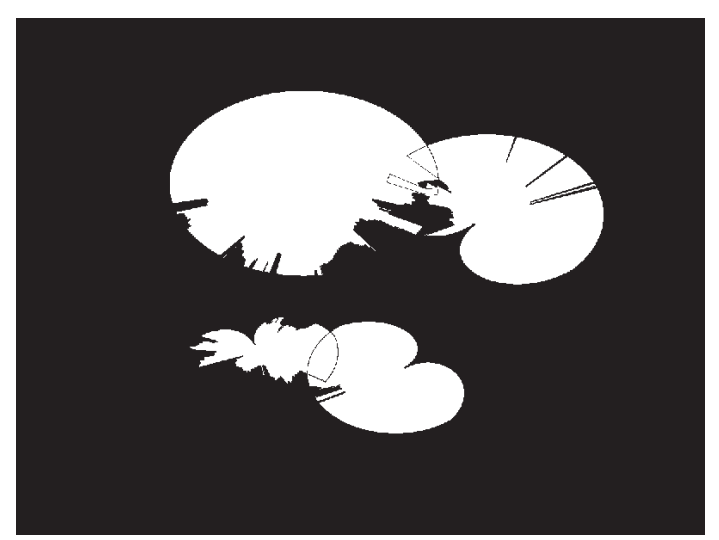

FIGURE 11: The binary image of suppression effect for each jamming aircraft against the enemy radar network effect obtained by MOPSO.

enemy radar network to be minimum and the minimum width of security zone to be maximum, that is, more focused on the suppression interference effect of the enemy radar network. So the optimal MACSIA scheme obtained by the improved MOPSO algorithm which also is called scheme one is shown in Figure 10. It can be seen from Figure 10 that the three jamming aircrafts are closer to the center of enemy radar network. The binary image of suppression effect for each jamming aircraft against the enemy radar network effect in scheme one is shown in Figure 12. The minimum width of security zone obtained by the improved MOPSO algorithm is $45 \mathrm{~km}$. Figure 9 is the optimal MACSIA scheme obtained by MOSPO under the same condition and Figure 11 is the binary image of suppression effect. The minimum width of security zone obtained by MOPSO is $41 \mathrm{~km}$ which is less than that obtained by the improved MOPSO algorithm. So it is proved that the improved MOPSO algorithm is better than MOPSO algorithm.

Secondly, when the decision maker needs the sum of the distance between each jamming aircraft and the center of 


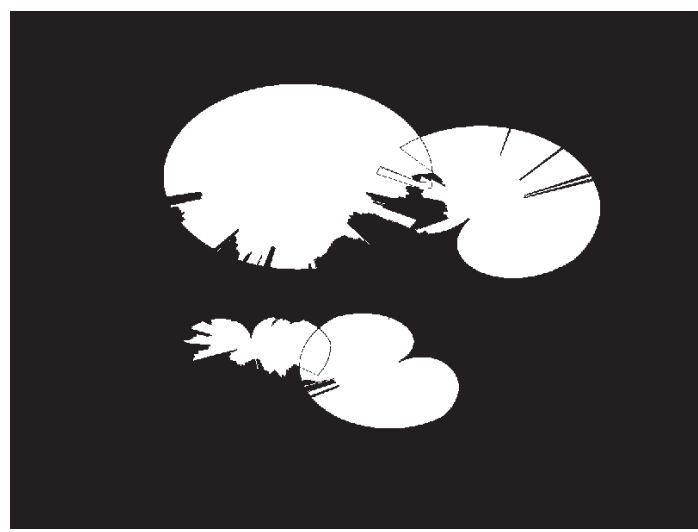

FIGURE 12: The binary image of suppression effect for each jamming aircraft against the enemy radar network effect in scheme one obtained by improved MOPSO.

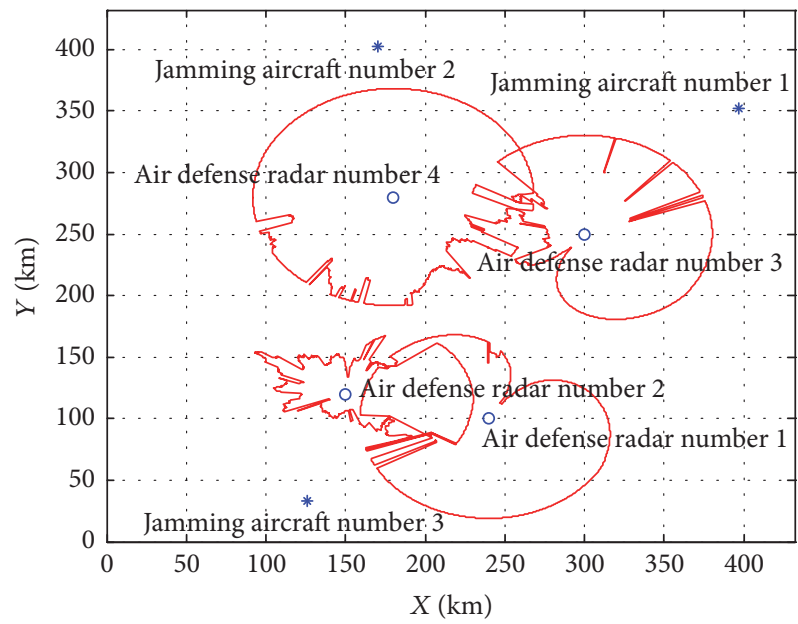

FIgURE 13: The optimal MACSIA scheme of the jamming aircrafts under the maximum total distance and minimum security zone minimum width obtained by MOPSO.

enemy radar network to be maximum and the minimum width of security zone to be minimum, that is, more focused on the safety of each jamming aircraft itself. So the optimal MACSIA scheme obtained by the improved MOPSO algorithm which also is called scheme two is shown in Figure 14. It can be seen from Figure 14 that the three jamming aircrafts are farther away from the center of enemy radar network. The binary image of suppression effect for each jamming aircraft against the enemy radar network effect in scheme two is shown in Figure 16. The minimum width of security zone obtained by the improved MOPSO algorithm is $28 \mathrm{~km}$. So the suppression interference effect of scheme two is worse than that of scheme one. Figure 13 is the optimal MACSIA scheme obtained by MOSPO under the same condition and Figure 15 is the binary image of suppression effect. The minimum width of security zone obtained by MOPSO is $24 \mathrm{~km}$ which is less than that obtained by the improved MOPSO algorithm. So it is proved that the improved MOPSO algorithm is better than MOPSO algorithm.

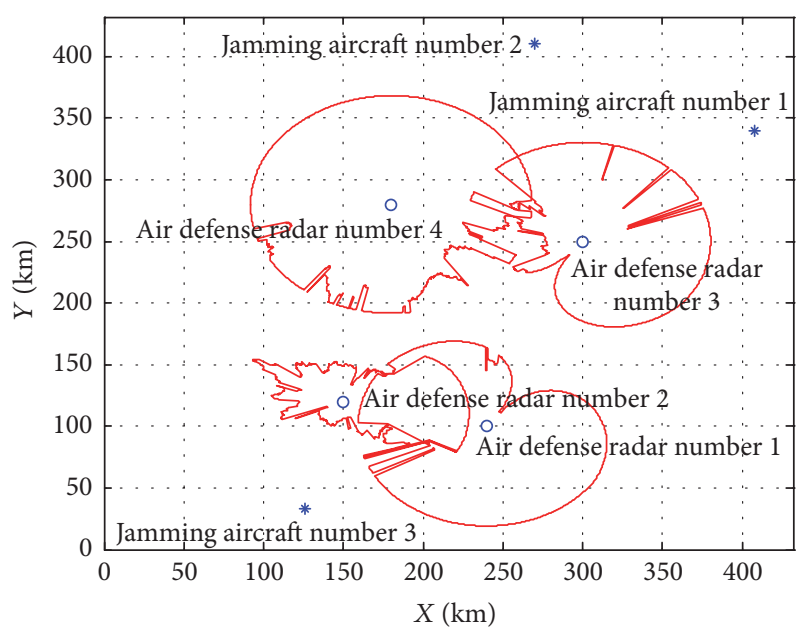

FIGURE 14: The optimal MACSIA scheme of the jamming aircrafts under the maximum total distance and minimum security zone minimum width obtained by improved MOPSO.

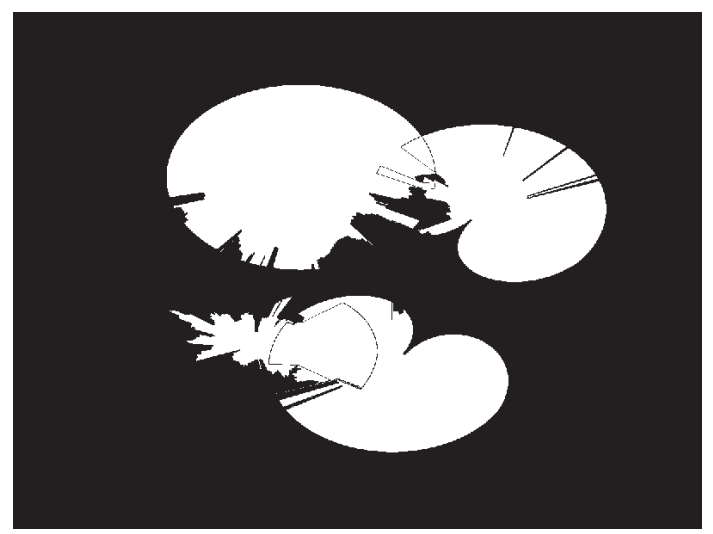

FIGURE 15: The binary image of suppression effect for each jamming aircraft against the enemy radar network effect obtained by MOPSO.

Thirdly, when the decision maker should consider not only the safety of the jamming aircrafts themselves, but also a good suppression interference effect. Then the optimal MACSIA scheme obtained by the improved MOPSO algorithm with moderate total distance and minimum width of security zone can be chosen which also is called scheme three; it is shown in Figure 18. The binary image of suppression effect for each jamming aircraft against the enemy radar network effect in scheme three is shown in Figure 20. The minimum width of security zone obtained by the improved MOPSO algorithm is $35.5 \mathrm{~km}$. So the suppression interference effect of scheme three is worse than that of scheme one but better than that of scheme two. Figure 17 is the optimal MACSIA scheme obtained by MOSPO under the same condition and Figure 19 is the binary image of suppression effect. The minimum width of security zone obtained by MOPSO is $32 \mathrm{~km}$ which is less than that obtained by the improved MOPSO algorithm. So it is proved that the improved MOPSO algorithm is better than MOPSO algorithm. 


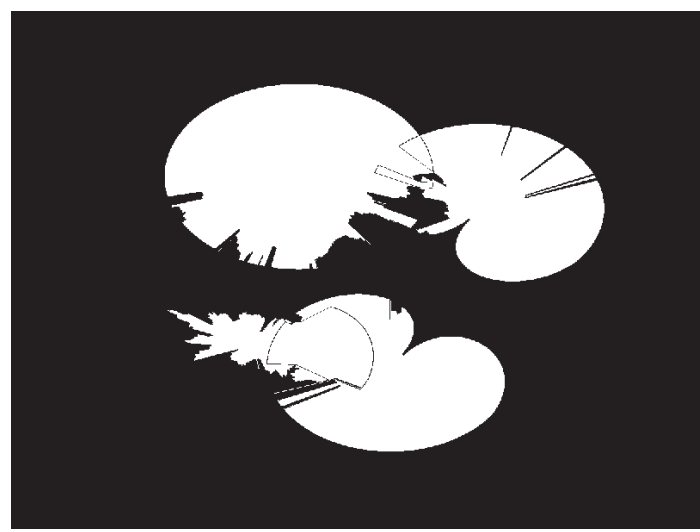

FIGURE 16: The binary image of suppression effect for each jamming aircraft against the enemy radar network effect in scheme two obtained by improved MOPSO.

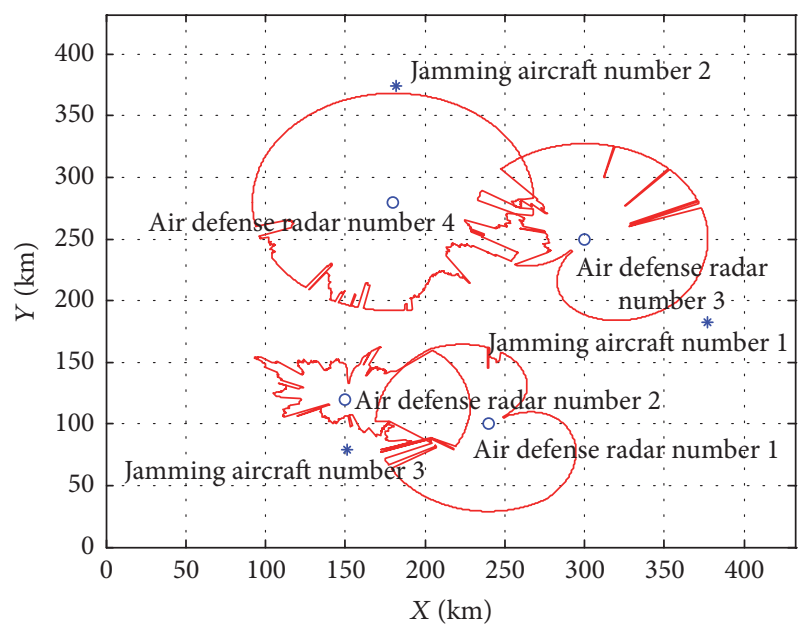

FIGURE 17: The optimal MACSIA scheme of the jamming aircrafts under the moderate total distance and moderate security zone minimum width obtained by MOPSO.

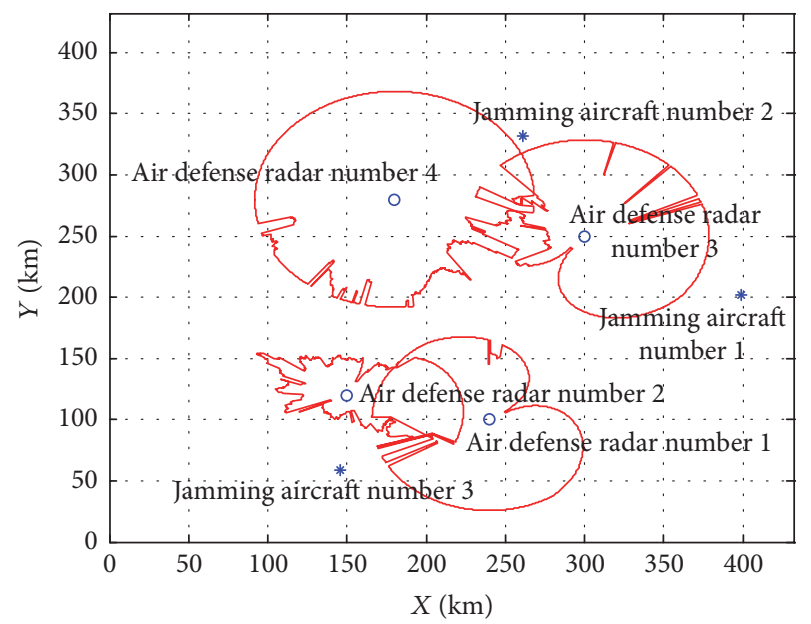

FIGURE 18: The optimal MACSIA scheme of the jamming aircrafts under the moderate total distance and moderate security zone minimum width obtained by improved MOPSO.

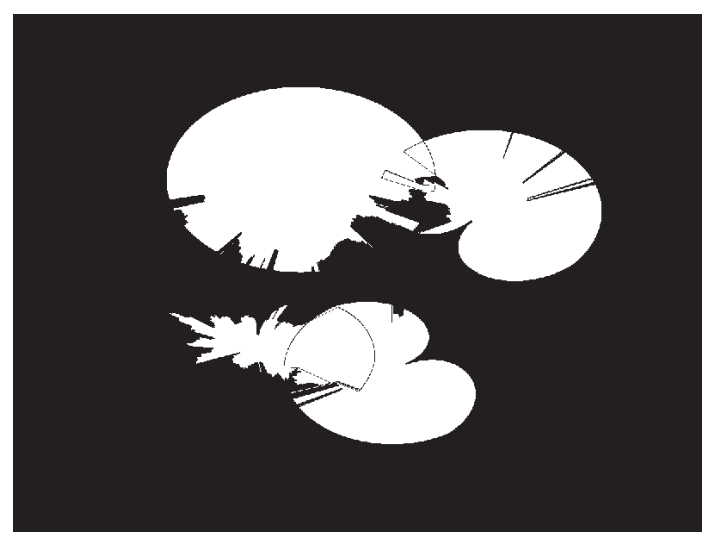

FIGURE 19: The binary image of suppression effect for each jamming aircraft against the enemy radar network effect obtained by MOPSO.

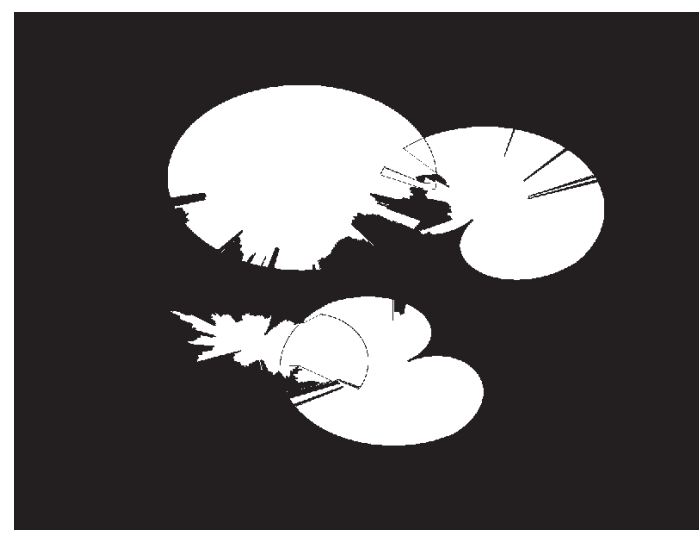

FIGURE 20: The binary image of suppression effect for each jamming aircraft against the enemy radar network effect in scheme three obtained by improved MOPSO.

It can be seen from the above that the three kinds of optimal MACSIA schemes of the jamming aircrafts obtained by the improved MOPSO algorithm have achieved the given suppression interference effect and formed the security zone that meets the given requirements.

\section{Conclusions}

(1) In this paper, for the problem of MACSIA in electronic warfare mission planning, the concept of route planning security zone is proposed, the solution to get the minimum width of security zone based on mathematical morphology is put forward, and the multiobjective optimization model of electronic interference array is built.

(2) The improved MOPSO algorithm is used to solve the model.

(3) Then the optimal MACSIA schemes can be obtained that meet the minimum width restriction of route planning security zone and the jamming aircrafts themselves are the most secure. Meanwhile, it verifies that the built model and the proposed improved MOPSO algorithm are feasible and effective. 
(4) The content of this paper is an important part of electronic warfare mission planning. It is the basis for subsequent penetration route planning which provides a safe and reliable planning space for the subsequent route planning. Therefore, this study has important practical significance.

When the battlefield environment changes, research on the problem of dynamic MACSIA has more practical significance. So we will take this as an entry point and explore more efficient and feasible dynamic MOPSO algorithm to solve the problem in the future work.

\section{Competing Interests}

The authors declare that there is no conflict of interests regarding the publication of this paper.

\section{Authors' Contributions}

Huan Zhang planned the work, completed the simulation experiment, and drafted the main part of the paper. Rennong Yang and Changyue Sun contributed to error analysis. Haiyan Han contributed to setup type.

\section{Acknowledgments}

The work described in this paper is partially supported by the Twelfth Five-Year Plan Preresearch Project (no. 402040401) and Science and Technology Research and Development Project of Shanxi Province (no. 2013kjxx-82).

\section{References}

[1] G. L. Fan, Z. M. Yang, and Y. Z. Wang, "Evaluation of four countering efficiency of netted radar based on multi-stage fuzzy synthetic judgment," Shipboard Electronic Countermeasure, vol. 36, no. 3, pp. 100-102, 2013.

[2] X. X. Zhang, "'Four countering' of radar in the early of the 21st century," Radar Science and Technology, vol. 1, no. 1, pp. 1-6, 2013.

[3] H. S. Shi, D. Li, Z. G. Zhao, S. Mao, and C. Shi, "Electronic jamming exercises influence on flight path planning of low observation aircraft," Journal of Nanjing University of Aeronautics and Astronautics, vol. 39, no. 2, pp. 154-158, 2007.

[4] Z.-J. Wang, X. Li, Q.-M. Zhou, and W.-L. Wang, "Optimal deployment of radar network based on multi-constrained GA," Xi Tong Gong Cheng Yu Dian Zi Ji Shu/Systems Engineering and Electronics, vol. 30, no. 2, pp. 265-268, 2008.

[5] S. J. Zhang, "Combat efficiency calculating method of long distance airborne jamming aircraft against ground early warning radar," Technology of Electronic Countermeasure, vol. 19, no. 3, pp. 29-31, 2004.

[6] M. Z. Ruan, H. J. Wang, Q. M. Li et al., "Efficiency evaluation of multi-jamming sources on stand-off jamming based on the exposed range," Systems Engineering and Electronics, vol. 31, no. 9, pp. 2110-2114, 2009.

[7] Z. Tang, X.-G. Gao, and Y. Zhang, "Research on the model evaluating the efficiency of the airborne active self-defense jamming system," Systems Engineering and Electronics, vol. 30, no. 2, pp. 236-239, 2008.
[8] Z.-Q. Chen, L. Yu, Y. Lu, and Z.-L. Zhou, "Research on optimized electronic warfare embattling countermining radar net," Acta Armamentarii, vol. 33, no. 1, pp. 89-94, 2012.

[9] C. A. Coello Coello, G. T. Pulido, and M. S. Lechuga, "Handling multiple objectives with particle swarm optimization," IEEE Transactions on Evolutionary Computation, vol. 8, no. 3, pp. 256-279, 2004.

[10] W. Hu, G. G. Yen, and X. Zhang, "Multiobjective particle swarm optimization based on Pareto entropy," Journal of Software, vol. 25, no. 5, pp. 1025-1050, 2014.

[11] M. G. Gong, Q. Cai, X. W. Chen, and L. Ma, "Complex network clustering by multiobjective discrete particle swarm optimization based on decomposition," IEEE Transactions on Evolutionary Computation, vol. 18, no. 1, pp. 82-97, 2014.

[12] M. G. Gong, L. C. Jiao, D. D. Yang, and W. P. Ma, "Research on evolutionary multi-objective optimization algorithms," Journal of Software, vol. 20, no. 2, pp. 271-289, 2009.

[13] G.-H. Hu, Z.-Z. Mao, and D.-K. He, "Multi-objective optimization for leaching process using improved two-stage guide PSO algorithm," Journal of Central South University of Technology, vol. 18, no. 4, pp. 1200-1210, 2011.

[14] R. S. Maciel, M. Rosa, V. Miranda, and A. Padilha-Feltrin, "Multi-objective evolutionary particle swarm optimization in the assessment of the impact of distributed generation," Electric Power Systems Research, vol. 89, pp. 100-108, 2012.

[15] Y.-Z. Luo and L.-N. Zhou, "Asteroid rendezvous mission design using multiobjective particle swarm optimization," Mathematical Problems in Engineering, vol. 2014, Article ID 823659, 13 pages, 2014.

[16] H. Xu, Y. Wang, and X. Xu, "Multiobjective particle swarm optimization based on dimensional update," International Journal on Artificial Intelligence Tools, vol. 22, no. 3, Article ID 1350015, 2013.

[17] Y. Cooren, M. Clerc, and P. Siarry, "MO-TRIBES, an adaptive multiobjective particle swarm optimization algorithm," Computational Optimization and Applications, vol. 49, no. 2, pp. 379400, 2011.

[18] L. D. S. Coelho, F. A. Guerra, and J. V. Leite, "Multiobjective exponential particle swarm optimization approach applied to hysteresis parameters estimation," IEEE Transactions on Magnetics, vol. 48, no. 2, pp. 283-286, 2012.

[19] Y. Zhang, D.-W. Gong, and Z. Ding, "A bare-bones multiobjective particle swarm optimization algorithm for environmental/economic dispatch," Information Sciences, vol. 192, pp. 213-227, 2012.

[20] F. Jolai, R. Tavakkoli-Moghaddam, and M. Taghipour, "A multiobjective particle swarm optimisation algorithm for unequal sized dynamic facility layout problem with pickup/drop-off locations," International Journal of Production Research, vol. 50, no. 15, pp. 4279-4293, 2012.

[21] S. M. Abd-Elazim and E. S. Ali, "Synergy of particle swarm optimization and bacterial foraging for TCSC damping controller design," WSEAS Transactions on Power Systems, vol. 8, no. 2, pp. 74-84, 2013.

[22] D. F. Zhang, Digital image processing with MATLAB, China Machine Press, Beijing, China, 2012.

[23] D. Liu, K. C. Tan, C. K. Goh, and W. K. Ho, "A multiobjective memetic algorithm based on particle swarm optimization," IEEE Transactions on Systems, Man, and Cybernetics, Part B: Cybernetics, vol. 37, no. 1, pp. 42-50, 2007. 
[24] J. Kennedy and R. Eberhart, "Particle swarm optimization," in Proceedings of the IEEE International Conference on Neural Networks, pp. 1942-1948, December 1995.

[25] N. Al Moubayed, A. Petrovski, and J. McCall, “ $D^{2}$ MOPSO: multi-objective particle swarm optimizer based on decomposition and dominance," in Proceedings of the 12th Europe Conference on Evolutionary Computation Combinatorial Optimization, pp. 75-86, Málaga, Spain, April 2012.

[26] Z. Li, R. Ngambusabongsopa, and E. Mohammed, "A novel diversity guided particle swarm multi-objective optimization algorithm," International Journal of Digital Content Technology and Its Applications, vol. 5, no. 1, pp. 269-278, 2011.

[27] C. A. Coello Coello and M. S. Lechuga, "MOPSO: a proposal for multiple objective particle swarm optimization," in Proceedings of the Congress on Evolutionary Computation (CEC '02), pp. 1051-1056, IEEE, Honolulu, Hawaii, USA, May 2002.

[28] J. Hazra and A. K. Sinha, "A multi-objective optimal power flow using particle swarm optimization," European Transactions on Electrical Power, vol. 21, no. 1, pp. 1028-1045, 2011.

[29] M. Daneshyari and G. G. Yen, "Cultural-based multiobjective particle swarm optimization," IEEE Transactions on Systems, Man, and Cybernetics, Part B: Cybernetics, vol. 41, no. 2, pp. 553567, 2011.

[30] X. Zhu, J. Zhang, and J. Feng, "Multiobjective particle swarm optimization based on PAM and uniform design," Mathematical Problems in Engineering, vol. 2015, Article ID 126404, 17 pages, 2015.

[31] Q. F. Zhang and H. Li, "MOEA/D: a multiobjective evolutionary algorithm based on decomposition," IEEE Transactions on Evolutionary Computation, vol. 11, no. 6, pp. 712-731, 2007.

[32] S.-J. Tsai, T.-Y. Sun, C.-C. Liu, S.-T. Hsieh, W.-C. Wu, and S.-Y. Chiu, "An improved multi-objective particle swarm optimizer for multi-objective problems," Expert Systems with Applications, vol. 37 , no. 8 , pp. 5872-5886, 2010. 


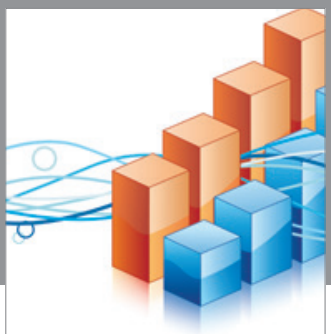

Advances in

Operations Research

vatem alat4

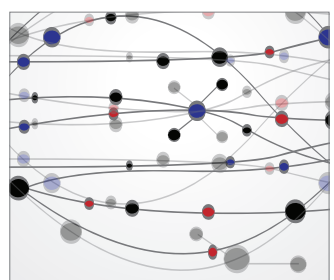

\section{The Scientific} World Journal
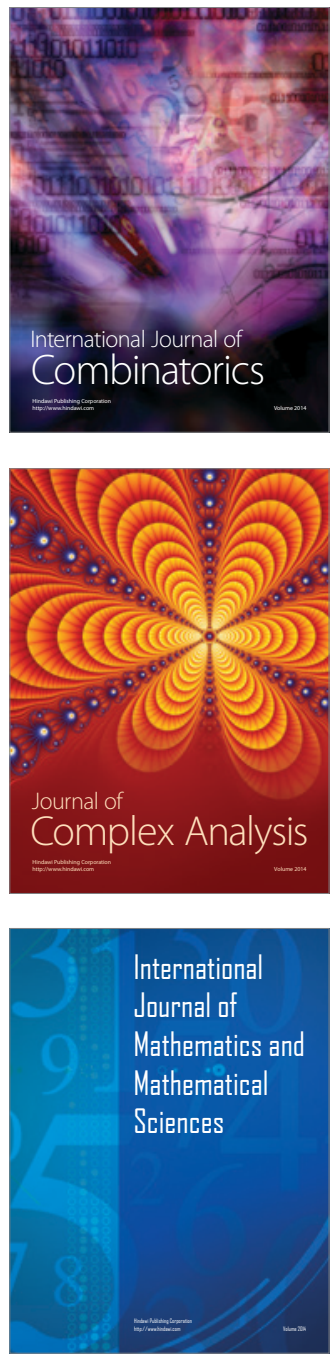
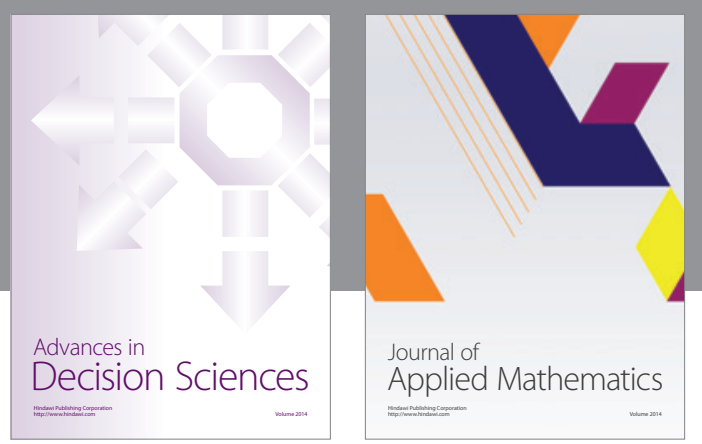

Algebra

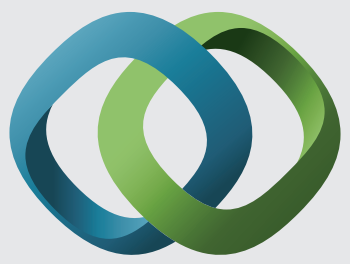

\section{Hindawi}

Submit your manuscripts at

https://www.hindawi.com
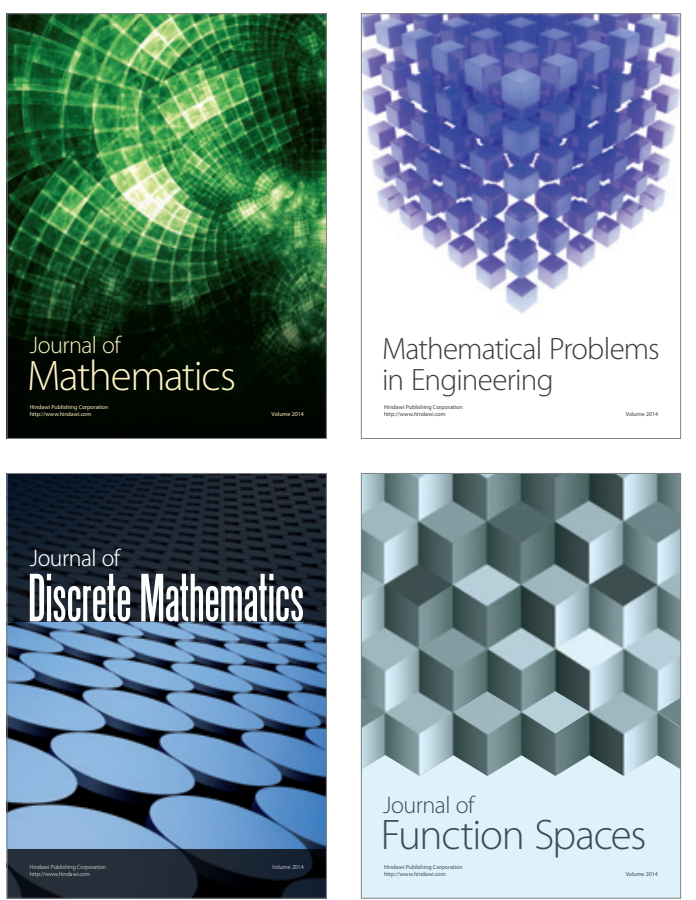

Mathematical Problems in Engineering
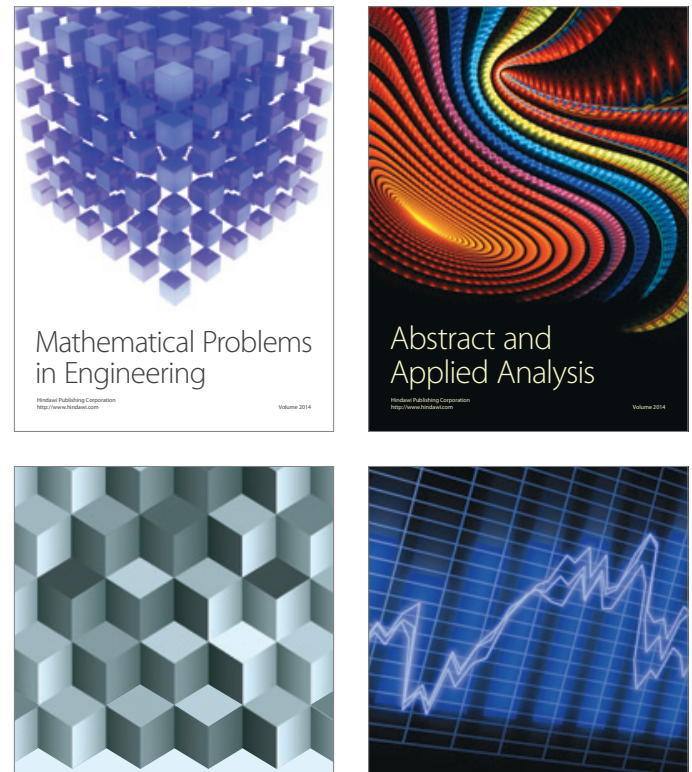

Journal of

Function Spaces

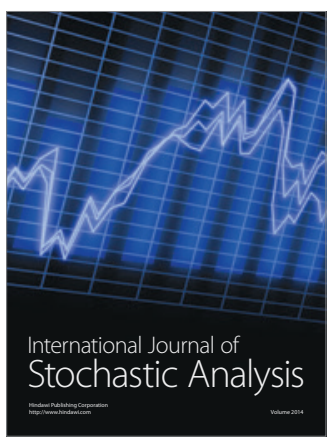

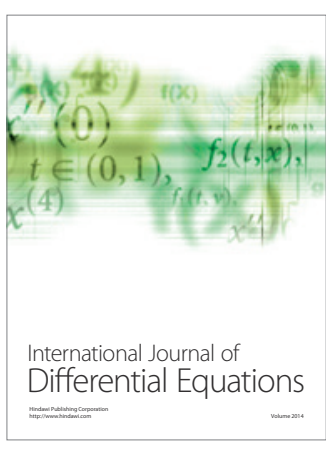
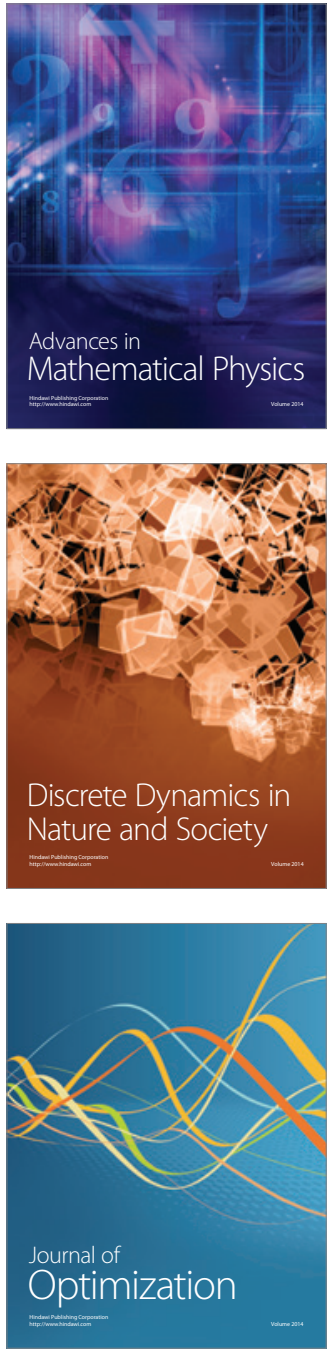